\title{
PROJECTIONS OR DEAD-ZONES: A NON-SINGULAR PERFORMANCE COMPARISON IN ROBUST ADAPTIVE CONTROL
}

\author{
Ahmad Sanei, Mark French \\ Department of Electronics and Computer Science \\ University of Southampton, U.K. \\ as99r|mcf decs.soton.ac.uk \\ http://www.isis.ecs.soton.ac.uk/control
}

Keywords: Robust Adaptive control, Non-singular Performance.

\begin{abstract}
We consider robust adaptive control designs for relative degree one, minimum phase linear systems of known high frequency gain. The designs are based on the dead-zone and projection modifications, and we compare their performance w.r.t. a worst case transient cost functional penalising the $\mathcal{L}^{\infty}$ norm of the output, control and control derivative. If a bound on the $\mathcal{L}^{\infty}$ norm of the disturbance is known, it is shown that the deadzone controller outperforms the projection controller when the a-priori information on the unknown system parameter is sufficiently conservative.
\end{abstract}

\section{Introduction}

It is well known that adaptive control is suitable for physical systems whose mathematical model contains an uncertain parameter $\theta$. A common feature of adaptive designs is the construction of a time varying parameter $\hat{\theta}$ whose value is controlled by an adaptive law. In contrast with most adaptive control mechanisms which would attempt to 'identify' or 'estimate' the uncertain parameter $\theta$ of the plant, the objective of a 'non-identifier-based' adaptive controller is to use certain information about the plant to find suitable methods of system regulation. In other words, the adaptive law has no interest to identify or estimate the unknown plant parameter $\theta$, but merely attempts to seek out a stabilising value of $\hat{\theta}$. See eg. $[1,6,7,9]$ and [3] for an overview.

However this method, like other adaptive controllers, is susceptible to phenomena such as parameter drift even when small disturbances are present. To overcome such problems, a number of standard techniques are widely utilised, such as deadzones, $\sigma$ modification, projection modification [8].

Each of these designs have advantages and drawbacks. For example, dead-zone modifications require a-priori knowledge of the disturbance level, and only achieve convergence of the output to some pre-specified neighbourhood of the origin (whilst keeping all signals bounded). In particular if the disturbance vanishes, then the dead-zone controller does not typ- ically achieve convergence to zero, the convergence remains to the pre-specified neighbourhood of the origin. On the other hand, projection modifications generally achieve boundedness of all signals, and furthermore have the desirable property that if no disturbances are present, then the output converges to zero, however, an arbitrarily small $\mathcal{L}^{\infty}$ disturbance can completely destroy any convergence of the output.

This illustrates that in the case of asymptotic performance, there are some known characterisations of 'good' and 'bad' behaviour. However, there are many situations in which we cannot definitively state whether a projection or dead-zone controller is superior even when only considering asymptotic performance. Furthermore, the known results, as with most results in adaptive control, are confined to singular performances, ie. without any consideration of the control signal.

In this paper we aim to compare the dead-zone and projection based adaptive controllers for finite dimensional minimum phase linear systems with relative degree one. The comparison has been made with respect to a worst case non-singular transient cost functional $\mathcal{P}$ penalising both the state $x$ and the input $u$ of the plant. We will identify a circumstance in which the dead-zone controller is superior to the projection controller with respect to $\mathcal{P}$.

\section{System and Basic Control Design}

Suppose $\Sigma$ is a SISO linear time invariant plant described by

$$
y=\frac{b_{m} s^{m}+b_{m-1} s^{m-1}+\cdots+b_{0}}{s^{n}+a_{n-1} s^{n-1}+\cdots+a_{0}}(u+d),
$$

where $a_{i}, b_{j}, 0 \leq i \leq n-1,0 \leq j \leq m$, are unknown constants and $d(\cdot)$ belongs to a class of bounded disturbances $\mathcal{D} \subset \mathcal{L}^{\infty}[0, \infty)$. We assume that only output $y(\cdot)$ is available for measurement. A minimal state space realisation of the plant in canonical observer form can be obtained as follows:

$$
\begin{aligned}
\Sigma\left(x_{0}, \theta, d(\cdot)\right): \quad \dot{x}(t) & =A x(t)+B(u(t)+d(t)), \\
y(t) & =C x(t) \\
x(0) & =x_{0}
\end{aligned}
$$


in which $x(t), B, C^{T} \in \mathbb{R}^{n}, A \in \mathbb{R}^{n \times n}$, and

$$
\begin{gathered}
A=\left[\begin{array}{ccccc}
-a_{n-1} & 1 & 0 & \ldots & 0 \\
-a_{n-2} & 0 & 1 & 0 & 0 \\
\vdots & \vdots & \vdots & \ddots & \vdots \\
-a_{1} & 0 & 0 & \ldots & 1 \\
-a_{0} & 0 & 0 & \ldots & 0
\end{array}\right], B=\left[\begin{array}{c}
0(\rho-1) \\
b_{m} \\
\vdots \\
b_{1} \\
b_{0}
\end{array}\right], \\
C=\left[\begin{array}{llll}
1 & 0 & \cdots & 0
\end{array}\right],
\end{gathered}
$$

where $\rho=n-m$ is the relative degree of the system and

$$
\theta=\left(a_{0}, \ldots, a_{n-1}, b_{0}, \ldots, b_{m}\right),
$$

represents the uncertain system parameters. We emphasise that by non-identifier-based control, we are not estimating unknown parameter $\theta$. Consider the following assumptions:

C1. The plant is minimum phase i.e. $b_{m} s^{m}+b_{m-1} s^{m-1}+$ $\cdots+b_{0}$ is Hurwitz.

$C 2$. The plant order $n$ is known; the plant is of relative degree one (i.e. $\rho=1$ ), and the high frequency gain is positive (i.e. $b_{m}=b_{n-1}>0$ ).

It was shown [1] that disturbance free $(\mathcal{D}=\{0\})$ systems of the form (2), i.e. $\Sigma\left(x_{0}, \theta, d(\cdot)\right)$ which satisfy $C 1, C 2$, are stabilised by the following simple adaptive high-gain controller:

$$
\begin{aligned}
\Xi: \quad u(t) & =-\hat{\delta}(t) y(t), \\
\dot{\hat{\delta}}(t) & =y(t)^{2} \quad \hat{\delta}(0)=0 .
\end{aligned}
$$

The above controller is a basis for 'non-identifier-based' adaptive controllers and $\hat{\delta}(\cdot)$ is called 'tuning function'. Special features of such controllers are their simplicity and the absence of any plant identification mechanism. For an early survey see [3].

\section{Robust Modifications to the Control Design}

It is well known that even a small $\mathcal{L}^{\infty}$ disturbance can cause a drift of the tunable function $\hat{\delta}(\cdot)$, see eg. [2]. To overcome this problem, two distinct approaches have been proposed: $(i)$ using an appropriate reference input, and (ii) modifying the adaptation law. In the next section we briefly explain two common methods in modifying the adaptive law i.e. dead-zone modification and parameter projection modification (see e.g. [8] for details).

\subsection{Dead-Zone Modification}

Consider unmodified adaptive law of the form $\dot{\hat{\delta}}(t)=$ $y(t)^{2}, \hat{\delta}(0)=0$. The idea of the dead-zone [10] is to modify the adaptive law so that the adaptive mechanism is "switched off' when system output $y(\cdot)$ lies inside a region $\Omega_{0}$ where the disturbance has a destabilising effect on the dynamics. The size of the disturbance is necessary a-priori knowledge in defining the dead zone. Let $d_{\max }$ be the a-priori knowledge of the upper bound of the disturbance level. For SISO output feedback systems (2), the dead-zone region $\Omega_{0}\left(d_{\max }\right)$ can be simply defined by $\Omega_{0}\left(d_{\max }\right)=\left[-\eta_{0}, \eta_{0}\right]$ where $\eta_{0}=\varrho\left(d_{\max }\right)$ and $\varrho: \mathbb{R}^{+} \rightarrow \mathbb{R}^{+}$. The standard definition of the modified adaptive law is $\dot{\hat{\delta}}=D_{\Omega_{0}\left(d_{\max }\right)}(y) y(t)^{2}$, where $D_{\Phi}(y):=0$ if $y \in \Phi$ and $D_{\Phi}(y):=1$, elsewhere. However, as an alternative - to avoid discontinuous switching, we use so-called 'smooth dead-zone' defined by

$$
D_{\Omega_{0}\left(d_{\max }\right)}^{\prime}(y)= \begin{cases}0, & y \in \Omega_{0}\left(d_{\max }\right) \\ |y|-\eta_{0}, & y \notin \Omega_{0}\left(d_{\max }\right)\end{cases}
$$

leading to the modified adaptive law of the form [3]

$$
\begin{aligned}
\Xi_{D^{\prime}}\left(d_{\max }\right): u(t) & =-\hat{\delta}(t) y(t)) \\
\dot{\hat{\delta}}(t) & =D_{\Omega_{0}\left(d_{\max }\right)}^{\prime}(y)|y(t)|, \hat{\delta}(0)=0, \\
\eta_{0} & =d_{\max },
\end{aligned}
$$

for which, the existence and uniqueness of the solution of the closed loop $\left(\Sigma\left(x_{0}, \theta, d(\cdot)\right), \Xi_{D^{\prime}}\left(d_{\max }\right)\right)$ follows directly from the classical theory of differential equations. The following theorem establishes the properties of such controllers:

Theorem 3.1. Consider the closed loop system $\left(\Sigma\left(x_{0}, \theta, d(\cdot)\right), \Xi_{D^{\prime}}\left(d_{\max }\right)\right)$ defined by (2), (7), where $C 1, C 2$ hold and $d(\cdot)$ is bounded. Assume that $d_{\max }$ is such that $\|d(\cdot)\|_{\mathcal{L}^{\infty}} \leq d_{\max }$. Then for any $x_{0} \in \mathbb{R}^{n}$, the following properties hold:

D1. There exist a unique solution $(x(\cdot), \hat{\delta}(\cdot)): \mathbb{R}^{+} \rightarrow \mathbb{R}^{(n+1)}$.

D2. $x(\cdot), \hat{\delta}(\cdot), u(\cdot)$ are uniformly bounded as a continuous function of $x_{0}, \theta, d_{\text {max. }}{ }^{1}$

D3. $y(t) \rightarrow \Omega_{0}$ as $t \rightarrow \infty$.

Proof. See [3] for the proof of D1,D3, and [11] for the proof of D2.

\subsection{Projection Modification}

The projection modification [4] is an alternative method to eliminate parameter drift by keeping the adaptive parameter within some a priori defined bounds. This can be accomplished by projecting the parameter estimator into a given compact convex set containing the true parameter vector. The general definition of the projection can be found in e.g. [5]. In our 'non-identifier-based' case, the definition is as follows: Define

$$
\delta_{\theta}=\inf \{\delta \geq 0 \mid A-\tilde{\delta} B C \text { is Hurwitz } \forall \tilde{\delta} \geq \delta\},
$$

and let $\delta_{\max }$ be a strict upper bound for $\delta_{\theta}$. Define the convex set $\Pi\left(\delta_{\max }\right):=\left[0, \delta_{\max }\right]$ and let $T_{m}$ be the first time instance that $\hat{\delta}$ hit the boundary $\delta_{\max }$ :

$$
T_{m}=\inf \left\{t \geq 0 \mid \hat{\delta}(t)=\delta_{\max }\right\} .
$$

\footnotetext{
${ }^{1}$ The function has domain $\mathbb{R}^{n} \times \mathcal{S} \times[0, \infty)$, where $\mathcal{S}:=\{\theta \mid$ $\Sigma\left(x_{0}, \theta, d(\cdot)\right)$ satisfies $\left.\mathrm{C} 1, \mathrm{C} 2\right\}$
} 
Then the projection controller is defined by:

$$
\begin{aligned}
& \Xi_{P}\left(\delta_{\max }\right): u(t)=-\hat{\delta}(t) y(t) \\
& \dot{\hat{\delta}}(t)=y(t)^{2}, \quad \hat{\delta}(0)=0, \quad \forall t \in\left[0, T_{m}\right], \\
& \hat{\delta}(t)=\delta_{\max }, \quad \forall t \in\left[T_{m}, \infty\right) .
\end{aligned}
$$

We denote the respective closed loop system by $\left(\Sigma\left(x_{0}, \theta, d(\cdot)\right), \Xi_{P}\left(\delta_{\max }\right)\right)$. The stability of the closed loop is examined in the following theorem.

Theorem 3.2. Consider the closed loop system $\left(\Sigma\left(x_{0}, \theta, d(\cdot)\right), \Xi_{P}\left(\delta_{\max }\right)\right)$ defined by (2), (10) where $C 1, C 2$ hold and $d(\cdot) \in \mathcal{L}^{\infty}$. Assume that $\delta_{\max }$ is such that $\delta_{\theta}<\delta_{\max }$. Then for any $x_{0} \in \mathbb{R}^{n}$, the following properties hold:

P1. The solution $(x(\cdot), \hat{\delta}(\cdot)): \mathbb{R}^{+} \rightarrow \mathbb{R}^{(n+1)}$ exist.

P2. $x(\cdot), \hat{\delta}(\cdot), u(\cdot)$ are uniformly bounded as a continuous function of $x_{0}, \theta,\|d\|, \delta_{\max }$.

Proof. See Theorem 4.3 in [11].

\section{Statement of the Main Results}

\subsection{Performance}

The ultimate goal in control theory is to design control laws which achieve good performance for any member of a specified class of systems. Consider a system $\Sigma \in \mathcal{S}$ that belongs to a set of all admissible systems. The performance of a controller $\Xi \in \mathcal{C}$ is measured by a cost functional $J$ of some measurable signals (state/output/input). The cost functional can be either singular $\left(J: \mathcal{X} \rightarrow \mathbb{R}^{+}\right)$, or non-singular $\left(J: \mathcal{X} \times \mathcal{U} \rightarrow \mathbb{R}^{+}\right)$, where $\mathcal{X}, \mathcal{U}$ are the function spaces representing the state and input signal spaces respectively.

Performance also can be measured in either the worst case or the average case. The worst case singular performance is formulated as a supremum of the cost functional over $\Upsilon$, where $\Upsilon$ is a set which contains all parameters (e.g. initial values, uncertainty, solutions of the closed loop, etc.) that distinguish one system from another:

$$
\mathcal{P}: P(\mathcal{S}) \times \mathcal{C} \rightarrow \mathbb{R}^{+}, \quad \mathcal{P}(\Sigma, \Xi)=\sup _{\Upsilon} J(\cdot),
$$

where $P(\mathcal{S})$ is the power set of $\mathcal{S}$.

As well as the above cases, two other classes of performance measure can be defined, namely asymptotic and transient performance. Roughly speaking, asymptotic performance shows the ultimate behaviour of a system, while transient performance monitors its behaviour in time. There is no specific definition for these costs and in general any measurement that satisfies above can be used as a cost function.

The goal of this paper is to establish a comparison between dead-zone and projection methods. We will compare the performances of the controllers with respect to the following worst case non-singular transient cost functional $\mathcal{P}\left(\Sigma\left(\mathcal{X}_{0}(\gamma), \Delta(\delta), \mathcal{D}(\epsilon)\right), \Xi\right)$, defined as follows:

$$
\begin{aligned}
& \mathcal{P}\left(\Sigma\left(\mathcal{X}_{0}(\gamma), \Lambda, \mathcal{D}(\epsilon)\right), \Xi\right) \\
& =\sup _{x_{0} \in \mathcal{X}_{0}(\gamma)} \sup _{\theta \in \Lambda} \sup _{d \in \mathcal{D}(\epsilon)}\left(\|x(\cdot)\|_{\mathcal{L}^{\infty}}+\|u(\cdot)\|_{\mathcal{L}^{\infty}}+\|\dot{u}(\cdot)\|_{\mathcal{L}^{\infty}}\right),
\end{aligned}
$$

where

$$
\begin{aligned}
\mathcal{X}_{0}(\gamma) & :=\left\{x_{0} \mid\left\|x_{0}\right\| \leq \gamma\right\}, \quad \gamma>0, \\
\mathcal{D}(\epsilon) & :=\left\{d(\cdot) \mid\|d(\cdot)\|_{\mathcal{L}} \leq \epsilon\right\}, \quad \epsilon \geq 0, \\
\Delta(\delta) & :=\left\{\theta \in \mathbb{R}^{2 n} \mid A-\delta B C \text { is Hurwitz and C1, C2 hold }\right\},
\end{aligned}
$$

and $\Lambda$ is any compact subset of $\Delta(\delta)$. There are elements on the boundary of $\Delta(\delta)$ which do not satisfy $\mathrm{C} 1, \mathrm{C} 2$ and for which the closed loop is not stable, hence generating an infinite cost. Therefore the second supremum cannot be taken over $\Delta(\delta)$.

\subsection{Main Result}

The following theorem is the main result of the paper:

Theorem 4.1. Consider the system $\Sigma\left(x_{0}, \theta, d(\cdot)\right)$ and the controllers $\Xi_{D^{\prime}}\left(d_{\max }\right)$ and $\Xi_{P}\left(\delta_{\max }\right)$ defined by (2), (7) and (10) respectively where $C 1, C 2$ hold. Let $\Lambda \subset \Delta(\delta)$ be compact. Consider the transient performance cost functional (12). Then $\forall d_{\max } \geq \epsilon, \exists \delta_{\max }^{*} \geq \delta$ such that $\forall \delta_{\max } \geq \delta_{\max }^{*}$,

$$
\begin{aligned}
\mathcal{P}\left(\Sigma\left(\mathcal{X}_{0}(\gamma), \Lambda, \mathcal{D}(\epsilon)\right), \Xi_{P}\left(\delta_{\max }\right)\right) \\
\quad>\mathcal{P}\left(\Sigma\left(\mathcal{X}_{0}(\gamma), \Lambda, \mathcal{D}(\epsilon)\right), \Xi_{D^{\prime}}\left(d_{\max }\right)\right)
\end{aligned}
$$

This theorem can be interpreted as stating that if the a-priori knowledge of the parametric uncertainty level $\delta_{\max }$ is sufficiently conservative $\left(\delta_{\max } \geq \delta_{\max }^{*}\right)$, then the dead-zone based design will out-perform the projection based design.

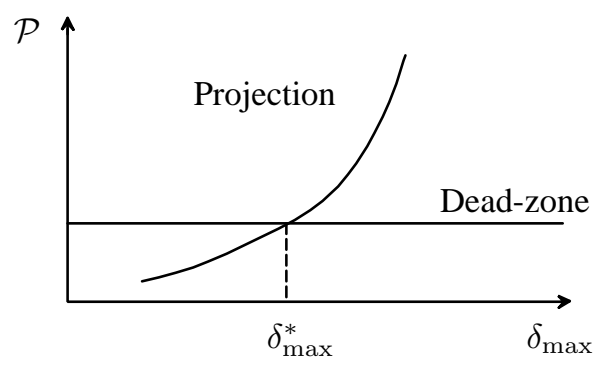

Figure 1: Statement of the main result

\section{Proof of Theorem 4.1}

Firstly, we show that $\mathcal{P}=\infty$ for the unmodified design (5), (Proposition 5.3). From this we can show that the projection modification design, $\Xi_{P}\left(\delta_{\max }\right)(10)$ has the property that $\mathcal{P} \rightarrow$ $\infty$ as $\delta_{\max } \rightarrow \infty$ (Proposition 5.4). Finally we show that $\mathcal{P}<$ $\infty$ for the dead-zone design, $\Xi_{D^{\prime}}\left(d_{\max }\right)$ (7) (Propositions 5.5). This suffices to establish Theorem 4.1. We do not give full 
details of the proof for brevity. The complete proof can be found in [11].

In the following, we frequently use the coordinate transformation matrices $S, S^{-1}$ defined by

$$
\begin{aligned}
& S:=\left[B(C B)^{-1}, T\right], \quad S^{-1}=\left[C^{T}, N^{T}\right]^{T}, \\
& N=\left[\left(b_{m-1} / b_{m} \ldots b_{0} / b_{m}\right)^{T} ; I_{(m-1)}\right] .
\end{aligned}
$$

where $T \in \mathbb{R}^{n \times(n-1)}$ is a basis matrix of $\operatorname{ker} C$. Observe that $S, S^{-1}$ depend continuously on $\theta$ over $\Delta(\delta)$, and

$$
\bar{x}(t):=\left(y(t), z(t)^{T}\right)^{T}=S^{-1} x(t),
$$

therefore

$$
\left[\begin{array}{c}
\dot{y}(t) \\
\dot{z}(t)
\end{array}\right]=\left[\begin{array}{cc}
\bar{a}_{1}-b_{m} k(t) & \bar{A}_{2} \\
\bar{A}_{3} & \bar{A}_{4}
\end{array}\right]\left[\begin{array}{c}
y(t) \\
z(t)
\end{array}\right]
$$

where $\bar{a}_{1} \in \mathbb{R}, \bar{A}_{2}^{T}, \bar{A}_{3} \in \mathbb{R}^{n-1}$ and $\bar{A}_{4} \in \mathbb{R}^{(n-1) \times(n-1)}$. Note that $\bar{a}_{1}-b_{m} k(t)<0, \forall t>t^{*}$ for sufficiently large $k^{*}$. It has been shown that $\bar{A}_{4}$ is stable [3], i.e. there exists a positive definite matrix $R=R^{T}>0$ satisfying the Lyapunov equation $R \bar{A}_{4}+\bar{A}_{4}^{T} R=-I_{n-1}$.

We also frequently use the compact notation $D(k):=A-$ $k B C$ for some $k>0$, and $\bar{D}:=S^{-1} D S$. Note that $\bar{D}\left(k^{*}\right)^{T} P+P \bar{D}\left(k^{*}\right) \leq-Q$, where the symmetric positive definite matrices $P, Q$ are defined as

$$
P=\left[\begin{array}{cc}
\frac{1}{2} & 0 \\
0 & R
\end{array}\right], \quad Q=\left[\begin{array}{cc}
1 & 0 \\
0 & \frac{1}{2} I_{n-1}
\end{array}\right]
$$

This can be shown by considering the Lyapunov function $V=$ $\bar{x}^{T} P \bar{x}$ and observing that

$$
\begin{aligned}
\dot{V} & =\bar{x}^{T}\left(\bar{D}(k)^{T} P+P \bar{D}(k)\right) \bar{x} \\
& \leq-\left(b_{m} k(t)-M\right) y(t)^{2}-\frac{1}{2}\|z(t)\|^{2} \leq \bar{x}^{T} Q \bar{x}
\end{aligned}
$$

for all $k>k^{*}$, where $M:=\left|\bar{a}_{1}\right|+\left(\left\|\bar{A}_{2}\right\|+2\|R\|\left\|\bar{A}_{3}\right\|\right)^{2} / 2$, and $k^{*}:=(M+1 / 2) / b_{m}$.

Lemma 5.1. Consider the system $\dot{z}=f(z)$ where $f$ is continuous. Then $\lim _{t \rightarrow \infty} z(t)=z^{*}$ implies that $z^{*}$ is an equilibrium point.

Proof. See Lemma 4.3 in [11].

Proposition 5.1. Consider the closed loop system $\left(\Sigma\left(x_{0}, \theta, d(\cdot)\right), \Xi\right)$ defined by (2), (5), where C1,C2 hold and $d(t)=\epsilon$ for some $\epsilon \neq 0$. Then

$$
\|x(t)\| \rightarrow 0 \text { as } t \rightarrow \infty \Longleftrightarrow \hat{\delta}(t) \rightarrow \infty \text { as } t \rightarrow \infty .
$$

Proof. $\rightarrow) \quad$ Suppose for contradiction $\hat{\delta}(t) \nrightarrow \infty$. Then $\hat{\delta}(t) \rightarrow \hat{\delta}^{*}<\infty$, since $\hat{\delta}(t)$ is monotonic by (5). Therefore $(x(t), \hat{\delta}(t))=\left(0, \hat{\delta}^{*}\right)$ is an equilibrium point of closed loop $\left(\Sigma\left(x_{0}, \theta, d(\cdot)\right), \Xi\right)$ by Lemma 5.1. Hence $\left(0, \hat{\delta}^{*}\right)$ must be a solution of the following equations:

$$
\begin{aligned}
x_{2}(t)-a_{n-1} x_{1}(t)+b_{m}\left(\epsilon-\hat{\delta}(t) x_{1}(t)\right) & =0, \\
\vdots & \\
-a_{0} x_{1}(t)+b_{0}\left(\epsilon-\hat{\delta}(t) x_{1}(t)\right) & =0, \\
x_{1}(t)^{2} & =0 .
\end{aligned}
$$

But $b_{0} \neq 0$ since system is minimum phase. We also have $\epsilon \neq 0$. Therefore $(x(t), \hat{\delta}(t))=\left(0, \hat{\delta}^{*}\right)$ cannot be a solution of (21), hence contradiction.

$\leftarrow$ ) Define the Lyapunov function $V(\bar{x}(t))=\bar{x}(t)^{T} P \bar{x}(t)$, where $\bar{x}(t), P$ are defined by (16) and (18) respectively. Denote $\bar{B}=S^{-1} B$ and $\bar{b}=\left(P+P^{T}\right) \bar{B}$. Define

$$
\begin{aligned}
\varphi(t):= & \bar{x}(t)^{T}\left(P \bar{D}\left(\hat{\delta}(t)-k^{*}\right)\right. \\
& \left.+\bar{D}\left(\hat{\delta}(t)-k^{*}\right)^{T} P\right) \bar{x}(t) .
\end{aligned}
$$

and note that as $\hat{\delta}(t) \rightarrow \infty$ we have $\varphi(t) \rightarrow-\infty$ for all $\bar{x}(t) \neq 0$. A routine calculation of the time derivative of $V(\bar{x}(t))$ implies:

$$
\begin{aligned}
\dot{V}(\bar{x}(t)) & \leq-\bar{x}(t)^{T} Q \bar{x}(t)+\bar{x}(t)^{T} \bar{b} \epsilon+\varphi(t), \\
& \leq-\underline{\lambda}(Q)\|\bar{x}(t)\|^{2}+\|\bar{x}(t)\||\bar{b}||\epsilon|+\varphi(t) .
\end{aligned}
$$

Applying Young's inequality to (24), we observe that $V(\cdot)$ is decreasing if

$$
\frac{\underline{\lambda}(Q)\|\bar{x}(t)\|^{2}}{2}-\varphi(t) \geq \frac{|\bar{b}|^{2}|\epsilon|^{2}}{2 \underline{\lambda}(Q)} .
$$

Now, we claim the convergence of $\bar{x}(\cdot)$ : if $\|\bar{x}(t)\| \not \rightarrow$ 0 as $t \rightarrow \infty$ then either 1 . $\liminf _{t \rightarrow \infty}\|\bar{x}(t)\|>0$ or 2 . $\liminf _{t \rightarrow \infty}\|\bar{x}(t)\|=0$ :

1. Suppose $\liminf _{t \rightarrow \infty}\|\bar{x}(t)\|>0$. Then there exists $\epsilon^{\prime}>$ 0 s.t. $\|\bar{x}(t)\|>\epsilon^{\prime} \forall t>0$. Since $\varphi(t) \rightarrow-\infty$ as $\hat{\delta}(t) \rightarrow \infty$, it follows by (23) that $\dot{V} \rightarrow-\infty$ as $t \rightarrow \infty$, i.e. $V \rightarrow-\infty$. This contradicts the positive definiteness of $V(\cdot)$.

2. If $\liminf _{t \rightarrow \infty}\|\bar{x}(t)\|=0$, then there must exists $\epsilon^{\prime}>0$, and a positive divergent sequence $\left\{t_{k}\right\}_{k \geq 1}$ such that $\dot{V}\left(\bar{x}\left(t_{k}\right)\right)>0$ and $\left\|\bar{x}\left(t_{k}\right)\right\|>\epsilon^{\prime}$. Since $\varphi\left(t_{k}\right) \rightarrow$ $-\infty$ as $k \rightarrow \infty$, it follows that (25) holds at time $t_{k}$, hence contradiction.

Therefore $\|\bar{x}(t)\| \rightarrow 0$ as $t \rightarrow \infty$; hence $x(t) \rightarrow 0$ by (16).

Proposition 5.2. Consider the closed loop system $\left(\Sigma\left(x_{0}, \theta, d(\cdot)\right), \Xi\right)$ defined by (2), (5), where C1,C2 hold and $d(t)=\epsilon$ for some $\epsilon \neq 0$. If $x(t)$ is uniformly continuous, then as $t \rightarrow \infty$

$$
\|x(t)\| \rightarrow 0, \quad \hat{\delta}(t) \rightarrow \infty
$$


Proof. Firstly we show that $y(t) \rightarrow 0$ as $t \rightarrow \infty$. From this we will prove that $\hat{\delta}(t) \rightarrow \infty$ and finally by Proposition 5.1, we conclude that $\|x(t)\| \rightarrow 0$ as $t \rightarrow \infty$. Suppose for contradiction $y(t) \nrightarrow 0$. Then there must exists a positive divergent sequence $\left\{t_{k}\right\}_{k \geq 1}$ for which $y\left(t_{k}\right) \geq M$ for some $M>0$. Since $x(t)$ is uniformly continuous, it follows that $y(t)$ is uniformly continuous, i.e. for $\epsilon=M / 2$

$$
\exists \omega>0 \text { s.t. } \forall \tau \in[0, \omega], \forall t>0,|y(t)-y(t+\tau)|<\frac{M}{2} .
$$

Therefore $\left|y\left(t_{k}\right)-y\left(t_{k}+\tau\right)\right|<M / 2$ and since $y\left(t_{k}\right) \geq M$, we have that $y\left(t_{k}+\tau\right)>M / 2$ i.e. $y(t) \geq M / 2$ for all $t \in\left[t_{k}, t_{k}+\right.$ $\omega]$. With no loss of generality, we may assume $t_{k+1}-t_{k} \geq \omega$. It follows that

$$
\hat{\delta}\left(t_{k}+\omega\right)=\int_{0}^{t_{k}+\omega} \dot{\hat{\delta}}(\tau) d \tau=\int_{0}^{t_{k}+\omega} y^{2}(\tau) d \tau \geq \frac{M^{2}}{4} k \omega,
$$

so $\hat{\delta}\left(t_{k}+\omega\right) \rightarrow \infty$ as $k \rightarrow \infty$. It follows by Proposition 5.1 that $\|x(t)\| \rightarrow 0$ as $t \rightarrow \infty$, therefore $y(t) \rightarrow 0$ by (2), hence contradiction.

Now we have $y(t)=x_{1}(t) \rightarrow 0$ and we claim $\hat{\delta}(t) \rightarrow \infty$. Suppose for contradiction $\hat{\delta}(t) \nrightarrow \infty$. Then $\hat{\delta}(t) \rightarrow \hat{\delta}^{*}<\infty$, since $\hat{\delta}(t)$ is monotonic by (5). Substitute this into (2), we have

$$
\begin{aligned}
\dot{x}_{1}(t) & =x_{2}(t)-\left(a_{n-1}+\hat{\delta}^{*} b_{m}\right) x_{1}(t)+b_{m} \epsilon, \\
\vdots & \\
\dot{x}_{n}(t) & =-\left(a_{0}+\hat{\delta}^{*} b_{0}\right) x_{1}(t)+b_{0} \epsilon,
\end{aligned}
$$

where by minimum phase property of system, $b_{i} \epsilon \neq 0, i \in$ $[0, m]$. As $x_{1}(t) \rightarrow 0$, equation (30) implies that $x_{n}(t) \rightarrow \infty$, since $x(\cdot)$ is uniformly continuous. It follows that $x_{n-1}(t) \rightarrow$ $\infty$, and cascading the argument yields to $x_{1}(t) \rightarrow \infty$ as $t \rightarrow$ $\infty$, hence contradiction. Therefore $\hat{\delta}(t) \rightarrow \infty$. From this and Proposition 5.1, the claim of the proposition follows.

Proposition 5.3. Consider the closed loop system $\left(\Sigma\left(x_{0}, \theta, d(\cdot)\right), \Xi\right)$ defined by (2), (5) where C1,C2 hold. Let $\Lambda \subset \Delta(\delta)$ be compact. Consider the transient performance cost functional (12). Then

$$
\mathcal{P}\left(\Sigma\left(\mathcal{X}_{0}(\gamma), \Lambda, \mathcal{D}(\epsilon)\right), \Xi\right)=\infty .
$$

Proof. Let $x_{0} \in \mathcal{X}_{0}(\gamma), \theta \in \Lambda$, and choose $d(t)=$ $\epsilon \neq 0$. Denote limsup by $\varlimsup$. Suppose for contradiction

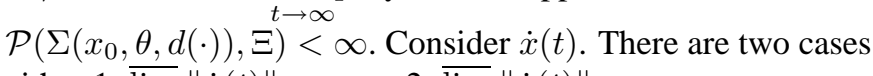
either $1 . \varlimsup \lim \|\dot{x}(t)\|=\infty$ or $2 . \varlimsup$

1. Suppose $\varlimsup_{\lim }\|\dot{x}(t)\| \quad=\quad \infty$, i.e. $\varlimsup\|A x(t)+B u(t)+B \epsilon\|=\infty$. Therefore either

(a) $\varlimsup \lim \|x(t)\|=\infty$, which implies that $\|x(\cdot)\|_{\mathcal{L}^{\infty}}=$ $\infty$, hence contradiction, or

(b) $\varlimsup \lim \|x(t)\|<\infty$, therefore $\varlimsup u(t)=\infty$ i.e. $\|u(\cdot)\|_{\mathcal{L}^{\infty}}=\infty$. Hence contradiction.
2. Suppose $\varlimsup\|\dot{l i m}\| \dot{x}(t) \|<\infty$ i.e. $x(\cdot)$ is uniformly continuous. Therefore by Proposition 5.2

$$
\|x(t)\| \rightarrow 0, \quad \hat{\delta}(t) \rightarrow \infty \quad \text { as } t \rightarrow \infty .
$$

Considering $\varlimsup \dot{\lim } \dot{u}(t)$, we observe that

$$
\begin{aligned}
\varlimsup \dot{\lim }(t)= & \varlimsup i m\left[-y(t)^{3}-\right. \\
& \hat{\delta}(t)(C A x(t)-C B(\hat{\delta}(t) y(t)-\epsilon))] .
\end{aligned}
$$

Note that $C B \neq 0$ since the relative degree $\rho=1$. Now there are two possible cases, either a) $\hat{\delta}(t) y(t) \not \epsilon$ (including the possibility that $\lim _{t \rightarrow \infty} \hat{\delta}(t) y(t)$ does not exist), or b) $\lim _{t \rightarrow \infty} \hat{\delta}(t) y(t)=\epsilon$

(a) Suppose $\lim _{t \rightarrow \infty} \hat{\delta}(t) y(t)$ does not exist or $\hat{\delta}(t) y(t) \not t \epsilon$ as $t \rightarrow \infty$. It follows by (32) that $\|\dot{u}(\cdot)\|_{\mathcal{L}^{\infty}}=\infty$; hence contradiction.

(b) Suppose $\lim _{t \rightarrow \infty} \hat{\delta}(t) y(t)=\epsilon$. By (32) we have that

$$
\forall \hat{\delta}^{*}>0 \exists T>0 \text { s.t. } \forall t>T \quad \hat{\delta}(t)>\hat{\delta}^{*} .
$$

Now we choose $d_{2}(t):=\epsilon, \quad \forall t \leq T, d_{2}(t):=$ $-\epsilon, \quad \forall t>T$. Note that $d_{2}(t)=d(t)$ for all $t \leq$ $T$. With this choice, by continuity and causality, we have that

$$
\lim _{t \rightarrow T^{+}} x(t)=x(T), \quad \lim _{t \rightarrow T^{+}} \hat{\delta}(t)=\hat{\delta}(T)
$$

where $\lim _{t \rightarrow T^{+}}$denote $\lim _{t \rightarrow T, t>T}$. It follows that

$$
\left(\lim _{t \rightarrow T^{+}} \dot{u}(t)\right)-\dot{u}(T)=2 \hat{\delta}(T) C B \epsilon \geq 2 \hat{\delta}^{*} b_{m} \epsilon .
$$

By choosing a suitable $\hat{\delta}^{*}$, it follows that $\hat{\delta}(T)$ can be made arbitrarily large and hence the difference (36) is arbitrarily large. Then either $\dot{u}(T)$ is large or $\lim _{t \rightarrow T^{+}} \dot{u}(t)$ is large, therefore $\|\dot{u}(\cdot)\|_{\mathcal{L}^{\infty}}$ can be made arbitrarily large. Hence contradiction.

Therefore at least one component of (12) diverges, hence

$$
\mathcal{P}\left(\Sigma\left(\mathcal{X}_{0}(\gamma), \Lambda, \mathcal{D}(\epsilon)\right), \Xi\right) \geq \mathcal{P}\left(\Sigma\left(x_{0}, \theta, d(\cdot)\right), \Xi\right)=\infty .
$$

Proposition 5.4. Consider the closed $\left(\Sigma\left(x_{0}, \theta, d(\cdot)\right), \Xi_{P}\left(\delta_{\max }\right)\right)$ defined by (2), (10) where $C 1, C 2$ hold. Let $\Lambda \subset \Delta(\delta)$ be compact. Consider the transient performance cost functional (12). Then

$$
\mathcal{P}\left(\Sigma\left(\mathcal{X}_{0}(\gamma), \Lambda, \mathcal{D}(\epsilon)\right), \Xi_{P}\left(\delta_{\max }\right)\right) \rightarrow \infty \quad \text { as } \quad \delta_{\max } \rightarrow \infty .
$$

Proof. It is convenient to define

$$
\begin{aligned}
\mathcal{P}_{[0, T]} & \left(\Sigma\left(x_{0}, \theta, d(\cdot)\right), \Xi\right) \\
& =\left(\|x(\cdot)\|_{\mathcal{L}^{\infty}[0, T]}+\|u(\cdot)\|_{\mathcal{L}^{\infty}[0, T]}+\|\dot{u}(\cdot)\|_{\mathcal{L}^{\infty}[0, T]}\right)
\end{aligned}
$$


Now let $M>0$. By Proposition 5.3 there exists $x_{0} \in \mathcal{X}_{0}$, $d(\cdot) \in \mathcal{D}(\epsilon), \theta \in \Lambda$ so that

$$
\mathcal{P}_{[0, \infty)}\left(\Sigma\left(x_{0}, \theta, d(\cdot)\right), \Xi\right) \geq 2 M
$$

It follows that $\exists T>0$ s.t. $\mathcal{P}_{[0, T]}\left(\Sigma\left(x_{0}, \theta, d(\cdot)\right), \Xi\right) \geq M$. Since $\delta_{\max }$ diverges, by choosing $\delta_{\max }=2 \hat{\delta}(T)$, we have that $\delta_{\max }>\hat{\delta}(T)$, i.e. the unmodified and the projection designs are identical on $[0, T]$, therefore

$$
\begin{aligned}
& \mathcal{P}\left(\Sigma\left(\mathcal{X}_{0}(\gamma), \Lambda, \mathcal{D}(\epsilon)\right), \Xi_{P}\left(\delta_{\max }\right)\right) \\
& \quad \geq \mathcal{P}_{[0, T]}\left(\Sigma\left(x_{0}, \theta, d(\cdot)\right), \Xi_{P}\left(\delta_{\max }\right)\right) \geq M .
\end{aligned}
$$

Since this holds for all $M>0$, this completes the proof.

Proposition 5.5. Consider the closed loop $\left(\Sigma\left(x_{0}, \theta, d(\cdot)\right), \Xi_{D^{\prime}}\left(d_{\max }\right)\right)$ defined by (2), (7) where $C 1, C 2$ hold. Let $\Lambda \subset \Delta(\delta)$ be compact. Consider the transient performance cost functional (12). Then

$\mathcal{P}\left(\Sigma\left(\mathcal{X}_{0}(\gamma), \Lambda, \mathcal{D}(\epsilon)\right), \Xi_{D^{\prime}}\left(d_{\max }\right)\right)<\infty, \quad \forall d_{\max }>\epsilon$.

Proof. Let $x_{0} \in \mathcal{X}_{0}(\gamma), \theta \in \Lambda$ and $d \in \mathcal{D}(\epsilon)$. A direct application of Property P2 of Theorem 3.1 guarantees the uniformly boundedness of $x(\cdot), \hat{\delta}(\cdot), u(\cdot)$ as a continuous function of $V^{*}\left(x_{0}, \theta, d_{\max }\right)$. It follows that

$$
\begin{aligned}
\dot{u}(t)=- & D_{\Omega_{0}}^{\prime}|y(t)| y(t)^{2} \\
& -\hat{\delta}(t) C((A-\hat{\delta}(t) B C) x(t)+B d(t)),
\end{aligned}
$$

is uniformly bounded in terms of a continuous function of $V^{*}\left(x_{0}, \theta, d_{\max }\right)$. Therefore

$$
\mathcal{P}\left(\Sigma\left(x_{0}, \theta, d(\cdot)\right), \Xi_{D^{\prime}}\left(d_{\max }\right)\right) \leq M\left(V^{*}\left(x_{0}, \theta, d_{\max }\right)\right),
$$

for some continuous $M\left(V^{*}\left(x_{0}, \theta, d_{\max }\right)<\infty\right.$. Taking the supremum over system parameters $x_{0}, \theta, d$ implies that for all $d_{\max } \geq \epsilon$,

$$
\begin{aligned}
& \mathcal{P}\left(\Sigma\left(\mathcal{X}_{0}(\gamma), \Lambda, \mathcal{D}(\epsilon)\right) \Xi_{D^{\prime}}\left(d_{\max }\right)\right) \\
& \quad \leq \sup _{x_{0} \in \mathcal{X}_{0}(\gamma)} \sup _{\theta \in \Lambda} \sup _{d \in \mathcal{D}(\epsilon)} M\left(V^{*}\left(x_{0}, \theta, d_{\max }\right)\right)<\infty .
\end{aligned}
$$

Proof of Theorem 4.1.

This is a simple consequence of Proposition 5.4 and Proposition 5.5.

\section{Conclusion}

In this paper we have established a rigourous result which demonstrate a situation in which we can compare the transient performance of projection and dead-zone based controllers of non identifier based adaptive designs. There are a number of directions in which the result can be generalised, for example: generalisation of the result for higher relative degrees and establishing whether the same results can be given for the alternative costs, for example, $\mathcal{P}=\|x(\cdot)\|_{\mathcal{L}^{\infty}}+\|u(\cdot)\|_{\mathcal{L}^{\infty}}$. Similarly we have developed results to demonstrate the contrary relationship between the controllers, ie. the results which show when the projection controllers outperform the dead-zone controllers, [11].

\section{References}

[1] C. I. Byrnes and J. C. Willems. Adaptive stabilization of multivariable linear systems. In Proc. 23rd IEEE Conference on Decision and Control, pages 1574-1577, 1984.

[2] B. Egardt. Stability of adaptive controllers. Lecture notes in Control and Information Sciences, Springer-Verlag, New York, 1979.

[3] A. Ilchmann. Non-Identifier-Based High-Gain Adaptive Control. Lecture notes in Control and Information Sciences, Springer-Verlag, London, 1993.

[4] G. Kreisselmeier and K. S. Narendra. Stable model reference adaptive control in the presence of bounded disturbances. IEEE Trans. on Automatic Control, 27(6):1169$1175,1982$.

[5] M. Krstić, I. Kanellakopoulos, and P. Kokotović. Nonlinear and Adaptive Control Design. John Wiley \& Sons, New York, 1995.

[6] B. Mårtensson. The order of any stabilizing regulator is sufficient a priori information for adaptive stabilization. Systems and Control Letters, 6:87-91, 1985.

[7] A. S. Morse. An Adaptive Control for Globally Stabilizing Linear Systems with Unknown High-frequency Gain. Springer-Verlag, Berlin, 1984.

[8] K. S. Narendra and A. M. Annaswamy. Stable Adaptive Systems. Prentice-Hall, 1989.

[9] R. D. Nussbaum. Some remarks on a conjecture in parameter adaptive control. Systems and Control Letters, 3:243-246, 1983.

[10] B. B. Peterson and K. S. Narendra. Bounded error adaptive control. IEEE Trans. on Automatic Control, 27(6):1161-1168, 1982.

[11] A. Sanei. Towards a Performance Theory of Robust Adaptive Control. PhD thesis, Department of Electronics and Computer Science, University of Southampton, U.K., 2003. 